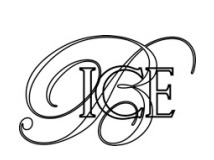

María Rodríguez de la Rúa*

Rosario Paradinas Zorrilla**

\title{
EL BANCO INTERAMERICANO DE DESARROLLO Y LA CELEBRACIÓN DE SU ASAMBLEA ANUAL EN BUSAN, COREA, EN MARZO DE 2015
}

El Banco Interamericano de Desarrollo, principal fuente de financiación multilateral para América Latina y el Caribe celebró su Asamblea Anual en la ciudad de Busan, en la República de Corea del Sur, el pasado mes de marzo de 2015. Durante esta Asamblea de Gobernadores se ha aprobado una Resolución por la cual se procede a aprobar el proceso de organización y capitalización de una nueva institución (NewCo), que agrupa las ventanillas del sector privado del Grupo BID. El objetivo principal es mejorar la efectividad de las intervenciones del Grupo BID con el sector privado de la región, a través del fortalecimiento de la cultura del sector privado y el establecimiento de una entidad más centrada estratégicamente y sometida a una mayor rendición de cuentas.

Palabras clave: instituciones y acuerdos internacionales, préstamos, sector privado.

Clasificación JEL: F33, F34.

\section{Introducción. EI BID}

El Banco Interamericano de Desarrollo (BID) es el banco regional más grande y más antiguo del mundo y la principal fuente de financiación multilateral para el desarrollo económico, social e institucional de América Latina y el Caribe. EI BID fue fundado en 1959 por un grupo de 20 países latinoamericanos y Estados Unidos. Más tarde se incorporaron los países del Caribe y Canadá. En 1974, la «Declaración de Madrid» permitió la entrada de socios extra regionales. A partir de ese año se fueron incorporando los países europeos,

\footnotetext{
* Directora Ejecutiva Alterna por España en el BID.

** Coordinadora de Área. Relaciones con del BID. Subdirección General de Instituciones Financieras Multilaterales.

Agradecimiento especial a Teresa Ramos y Paloma Ruiz por sus contribuciones.

Versión de junio de 2015.
}

entre ellos España, y Japón y en los últimos tiempos, se incorporaron la República de Corea del Sur en 2005 y la República Popular de China en 2009, lo que permitió dotar al Banco de un carácter más global que refleja, además, la intensificación en los últimos años de las relaciones entre Asia y América Latina. Reflejo de ello, ha sido la celebración de la última Asamblea Anual del Banco en la República de Corea del Sur.

EI BID cuenta en la actualidad con 48 países miembros: 26 países prestatarios latinoamericanos y caribeños, Estados Unidos, Canadá y 20 países extra regionales. Los países prestatarios de la región son los accionistas mayoritarios. Este ha sido el sello distintivo de este banco regional, un banco de desarrollo enfocado a las necesidades de la región y sentido por los países de América Latina y el Caribe como una institución propia. 
A lo largo del tiempo, el Banco ha ido adquiriendo algunas ventajas comparativas como son un sólido enfoque país; la diversificación de productos financieros y no financieros; el conocimiento de la región y su acompañamiento en los proyectos, así como su capacidad para apoyar el comercio y la integración regional entre otros.

EI BID también se ha ido adaptando a la evolución de la región y a sus ciclos económicos, mejorando sus instrumentos y su capacidad de respuesta a las cambiantes condiciones y necesidades de sus países prestatarios. En este sentido, se ha venido impulsando un proceso de reorganización interna del Banco o «realineamiento» con objeto de readaptar la institución a las nuevas realidades y dotarla de una mayor flexibilidad y capacidad de respuesta. Este proceso ha culminado en la Asamblea Anual, celebrada el pasado mes de marzo en la República de Corea del Sur, con la aprobación del proceso de capitalización de una Nueva Corporación que agrupará las ventanillas del sector privado. Este proceso de fusión de las ventanillas del sector privado va a ser el tema principal de nuestro artículo.

\section{El grupo BID en 2014}

En 2014 el banco aprobó un programa de 168 proyectos por un total de 13.843 millones de USD. Aunque el volumen total de aprobaciones fue ligeramente inferior al de 2013 , estos resultados consolidan la tendencia creciente del nivel de aprobaciones del Banco. En promedio, las aprobaciones anuales han crecido notablemente en los últimos cinco años comparados con los resultados del quinquenio anterior, elevándose de 9.750 millones de USD en el periodo 2005-2009, a 12.576 millones de USD en el periodo 2010-2014.

En 2014, el Banco continuó consolidándose como la principal fuente de financiación multilateral para América Latina y el Caribe, especialmente en cuanto apoyo para países de los grupos $C$ y D (países pequeños y vulnerables).
Las aprobaciones de préstamos en 2014 se concentraron en las cinco áreas prioritarias del marco de la novena ampliación de capital del BID. A nivel sectorial, el 42 por 100 de la financiación aprobada se dirigió a apoyo institucional para el desarrollo, el 38 por 100 a los sectores de infraestructura y medio ambiente, el 16 por 100 en programas para el sector social y el 5 por 100 a programas de integración y comercio exterior.

Respecto a actividades sin garantía soberana, el Banco aprobó 63 operaciones en 2014, por un volumen total de 2.823 millones de USD, lo cual representó el 20 por 100 del volumen de crédito. Adicionalmente, la Corporación Interamericana de Inversiones (CII) aprobó 64 proyectos (préstamos y transacciones de capital) por un total de 426,3 millones de USD, aumentado de esta forma su apoyo al crecimiento y al fomento de las pymes en la región. Además la CII movilizó 693,2 millones de USD por conducto de préstamos $B$ en el curso 2014. Por último, el Fondo Multilateral de Inversiones (FOMIN) aprobó 78 proyectos por 94,4 millones de USD, de los cuales 68 correspondieron a recursos no reembolsables de cooperación técnica y 10 fueron préstamos u operaciones de inversión combinadas con fondos no reembolsables.

Por destino de los préstamos, en 2014, no hubo variaciones significativas, con el Grupo A de países (economías más avanzadas, Argentina, Brasil, México y Venezuela) con un 46 por 100 del total de aprobaciones de préstamos del banco; el grupo B (economías medianas; Chile, Colombia y Perú) con un 18 por 100; el grupo C (Bahamas, Barbados, Costa Rica, Jamaica, Panamá, Suriname, Trinidad y Tobago y Uruguay) con un 10 por 100 y el grupo D (Belice, Bolivia, República Dominicana, Ecuador, El Salvador, Guatemala, Guayana, Haití, Honduras, Nicaragua y Paraguay) con un 26 por 100.

\section{España y el BID}

España ingresó en el BID, junto con otros países no regionales, en 1976. España detentaba el 1,896 por 100 del capital de la institución (la misma $\triangleright$ 


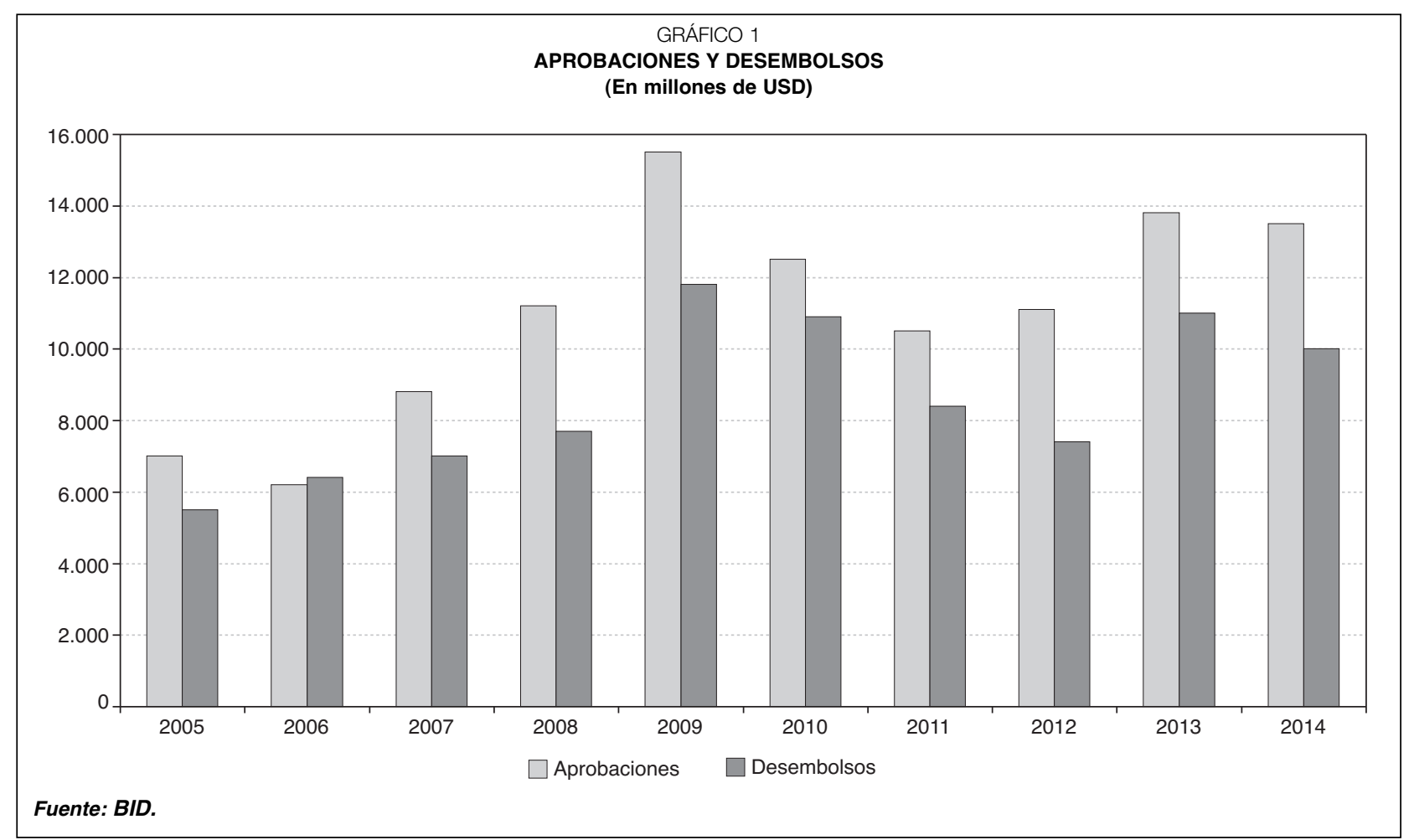

participación que Francia, Italia y Alemania), pero como resultado de la compra de la parte correspondiente de las acciones de Holanda de la Novena Ampliación de capital, España ha pasado a una participación del 1,965 por 100 del capital del Banco.

El Gobernador titular por España es el Ministro de Economía y Competitividad. España forma junto con Francia, Austria, Dinamarca, Finlandia, Noruega y Suecia una «Silla» o «circunscripción» a efectos de representación en el Directorio, disponiendo en total de 4,78 por 100 de los votos. Nuestro país ejerce con Francia de manera alterna cada tres años la Dirección Ejecutiva de la Silla.

EI BID es la institución financiera multilateral en la que trabajan proporcionalmente más españoles. Aproximadamente, un 5 por 100 del personal es español; más específicamente 253 españoles de los cuales 111 son funcionarios del Grupo BID y 142 son consultores.

En noviembre de 2010, España y el BID firmaron un Acuerdo Marco que constituye el actual marco de referencia básico para canalizar la actuación de nuestro país en el BID a través de la financiación de proyectos en áreas de mutuo interés y vinculados a la estrategia institucional del Banco, además de las actividades de cooperación técnica, y de la cesión de profesionales españoles en el BID.

Además de nuestra participación accionarial, España participa en algunos fondos en el que los que el grupo BID interviene como gestor fideicomisario, tesorero o presta apoyo administrativo de algún tipo. Estos fondos son los siguientes:

- Fondo Español del Agua: dotado con $790 \mathrm{mi}-$ llones de euros (MAEC), este Fondo financia proyectos en la región en el ámbito del agua y el saneamiento. En concreto, en las áreas de agua potable, alcantarillado, tratamiento de aguas residuales, drenaje de aguas pluviales urbanas, gestión de recursos hídricos, gestión de recursos sólidos y eficiencia y gestión operativa.

- Fondo General de Cooperación de España (FGCE): 63 millones de euros (MINECO). España mantiene en el BID uno de los fondos más importantes en el Banco. El FGCE se estableció en 2001 con una contribución inicial de 50 millones de euros, que provenían de la Cuenta de Compensación $\triangle$ 
del Fondo V Centenario. En el contexto de la firma del Acuerdo Marco de 2010 se hizo una contribución adicional al FGCE por un importe de $13 \mathrm{mi}-$ llones de euros. Este fondo apoya las operaciones de cooperación técnica en los sectores de modernización del Estado, integración regional y mejora de la competitividad, y financia también el programa de cesión de profesionales. Este fondo igualmente contempla la cofinanciación de proyectos de interés para España. Los recursos del FGCE también se han utilizado para realizar contribuciones a:

Fondo de desastres naturales (4 millones de euros).

Fondo de economía del conocimiento (2 millones de euros).

Fondo para la integración de la infraestructura regional (2 millones de euros).

Corporación Interamericana de Inversiones, CII (1,8 millones de USD) para programas de apoyo a las pymes y consultoría en general.

Fondo Multilateral de Inversiones, FOMIN (4,4 millones de euros) para el Programa de Apoyo en la Innovación y el Programa para promover las Asociaciones Público Privadas.

- Iniciativa Mesoamericana de Salud: 14 millones de USD (MAEC). Esta iniciativa es una asociación público-privada constituida entre el Gobierno de España, la Fundación Bill \& Melinda Gates, el Instituto Carlos Slim de la Salud (ICSS) y el BID como fiduciario, y está destinada a reducir las brechas de equidad en salud que enfrentan las poblaciones más pobres y vulnerables a través de actuaciones en las áreas de salud reproductiva; salud materna, neonatal e infantil; nutrición materna e infantil, vacunación, malaria y dengue.

- Fondo SECCI (Energía Sostenible y Cambio Climático): 10 millones de USD (MINECO). Esta Iniciativa del BID (SECCI, Sustainable Energy and Climate Change Iniciative) tiene por objeto apoyar al Banco en el desarrollo y el uso de fuentes de energía renovable, de tecnologías y aplicaciones de eficiencia energética, la financiación del mercado del carbono y la promoción de acciones en los ámbitos de adaptación y mitigación del cambio climático.
- Fondo Español de Empresariado Social: 10 millones de USD (MINECO). Este fondo financia instituciones privadas, con y sin fines de lucro, que busquen el desarrollo socio-económico de microempresarios y pequeños productores mediante actividades productivas y con efectos demostrativos en la provisión de servicios básicos.

- Fondo Regional de Tecnología Agropecuaria (FONTAGRO): 10 millones de euros (MAEC). Alianza de países establecida para financiar investigación e innovación en el sector agropecuario y constituye además un foro de análisis y discusión en ciencia y tecnología para el sector rural.

- Fondo V Centenario: 70 millones de USD (MINECO). Establecido en 1990, este fondo ha servido para financiar dos operaciones, un préstamo a la Universidad Autónoma de México y el proyecto SIEPAC (Sistema de Interconexión Eléctrica de los países de América Central). En la actualidad este fondo está generando reembolsos que se están traspasando al FGCE.

\section{El proceso de fusión de las ventanillas del sector privado del BID}

\subsection{Antecedentes}

El Grupo BID apoya el desarrollo mediante el sector privado. Con este fin provee financiación con y sin garantía soberana como también productos no financieros.

Como hemos visto, la actividad del BID, en cuanto a nivel de préstamos sin garantía soberana en 2014, representó el 20 por 100 de su actividad. No obstante, el proceso de reorganización interna actual, que vamos a proceder a explicar, tiene como principal objetivo impulsar la actividad del sector privado del Banco sin menoscabar la actividad con el sector público.

La actividad del BID de apoyo al sector privado de la región se realiza hasta el momento a través de 4 ventanillas: el Departamento de Financiación Estructurada o Financiamiento Estructurado y Corporativo (SCF) ${ }^{1}$; la Corporación Interamericana de $\triangleright$

\footnotetext{
1 Dentro del BID que ofrece apoyo al sector privado de la región en los ámbitos de mercados de capital, comercio e infraestructuras.
} 
Inversiones $(\mathrm{CII})^{2}$; el Fondo Multilateral de Inversiones (FOMIN) ${ }^{3}$; y la Iniciativa de Oportunidades para la Mayoría $(\mathrm{OMJ})^{4}$. Estas ventanillas están localizadas en la actualidad en dos instituciones legalmente independientes y en un fondo fiduciario. Esta fragmentación institucional se refleja en distintas estructuras de gobernanza, balances contables y modelos operativos, así como en una superposición de mandatos. A estas ventanillas habría que añadir las operaciones con garantía soberana que se dirigen a apoyar el desarrollo del sector privado en los países.

La evaluación intermedia realizada por la Oficina de Evaluación (OVE) en el contexto de la revisión del Noveno Aumento de Capital, destacó la falta de coordinación entre las áreas operativas con y sin garantía soberana y entre las distintas ventanillas del sector privado, con mandatos superpuestos especialmente en los ámbitos de los mercados financieros y de micro, pequeñas y medianas empresas, para desarrollar la actividad en el ámbito del desarrollo del sector privado de la región. En respuesta a esta evaluación, en la Asamblea Anual de Gobernadores de Panamá, de marzo de 2013, se acordó la creación de un Comité Ad Hoc ${ }^{5}$, para estudiar una reforma de la actividad del Banco en el ámbito del sector privado.

En la reunión del Comité de la Asamblea de Gobernadores celebrada en octubre de 2013, en Washington, se presentaron a los Gobernadores varios escenarios en torno a dos grandes alternativas de estructura de las actividades del BID con el sector privado: una estructura de fusión hacia dentro donde todas las actividades se consolidaban operativa y financieramente dentro del BID, o una estructura de fusión hacia afuera en la que el BID centraría su labor en las actividades con

\footnotetext{
2 Institución jurídicamente independiente del BID, que concede financiación a empresas privadas de América Latina y el Caribe, con especial atención a las pymes.

${ }^{3}$ Instrumento del BID creado con el propósito de buscar formas innovadoras de apoyo a la inversión privada y mejorar el entorno empresarial apoyando a la micro y pequeña empresa.

${ }^{4}$ Promueve y financia modelos de negocio sostenibles que involucran a compañías del sector privado, gobiernos locales y comunidades en el desarrollo y provisión de productos y bienes de calidad a la población de bajos ingresos.

5 Formado por representantes del Directorio del BID y de la CII y del Comité de donantes del FOMIN.
}

garantía soberana y todas las actividades del grupo sin garantía soberana se consolidan operativa y financieramente en una «Nueva Corporación» a partir de la actual CII. Los Gobernadores, con el apoyo mayoritario de los países de América Latina, respaldaron la opción de fusión hacia afuera.

De esta forma, durante la Asamblea Anual del BID de 2014 en Salvador de Bahía (Brasil), se aprobó finalmente una resolución respaldando la consolidación operativa y financiera de las actividades con el sector privado en esta nueva entidad, (el FOMIN sería parte de esta consolidación pero mantendría su gestión independiente). Se consideró que la consolidación dentro de la CII, en vez de crear una nueva entidad, tiene varias ventajas desde el punto de vista legal. Ningún cambio a los Convenios Constitutivos del BID o de la Cll sería necesario para implementar la Fusión hacia afuera y la Nueva Corporación podría operar dentro del Marco Operativo y de gestión establecido en el Convenio Constitutivo actual de la CII. Esta resolución contemplaba también que se presentaría para decisión de los Gobernadores una propuesta de capitalización para la entidad consolidada, no más tarde de la Asamblea 2015, teniendo a su vez en cuenta la nueva Política de Adecuación de Capital (PAC) ${ }^{6}$.

Durante la reunión del Comité de la Asamblea de Gobernadores celebrada en octubre de 2014 en Washington, los Gobernadores solicitaron que prosiguieran los trabajos en relación con la propuesta de organización, de capitalización, y el plan de implementación, todo lo cual se presentaría a los Gobernadores en la Reunión Anual del BID de Busán. Durante la reunión se expusieron las implicaciones financieras, de gobernabilidad, coordinación y fortalecimiento del marco de efectividad en el desarrollo. Se presentaron los pilares y áreas prioritarias de intervención y una serie simulaciones financieras en torno a la estructura de fusión hacia afuera.

6 El mandato de la PAC de preservar la calificación AAA del BID fue aprobado en octubre de 2014. El reglamento que rige la implementación de la PAC se aprobó por los Gobernadores en marzo de 2015. 
La puesta en marcha de la fusión hacia afuera requiere de un periodo de tiempo en que se practique la contabilidad cruzada hasta que todas las operaciones sin garantía soberana se consoliden en la Nueva Corporación. De las simulaciones financieras de la fusión hacia afuera se concluía que sin nuevo capital adicional, la Nueva Corporación no podría aumentar su capacidad de préstamo actual de manera significativa y, por lo tanto, habría muchas limitaciones para desarrollar la visión renovada tal y como estaba planteada.

\subsection{Visión renovada del Grupo BID}

La visión renovada del grupo del $\mathrm{BID}^{7}$ para promover el desarrollo a partir del sector privado establece que la Nueva Corporación tiene dos objetivos principales: el fortalecimiento de una cultura del sector privado y el establecimiento de una entidad más centrada estratégicamente y sometida a una mayor rendición de cuentas.

La visión renovada se ha construido a partir de las ventajas comparativas del grupo BID y sobre tres pilares:

La selectividad estratégica en áreas que marquen una mayor diferencia y dónde se maximice el impacto.

Enfoque sistémico para asegurar que los recursos tengan un impacto mayor que el de un proyecto individual.

Un enfoque ex-ante y ex-post sobre la efectividad en el desarrollo.

La visión renovada establece además cinco Áreas Prioritarias de Intervención (API):

API 1. Aumentar el acceso de las mypymes a la financiación y a la asistencia técnica,

API 2. Promover la infraestructura para el desarrollo.

API 3. Apoyar la innovación y el desarrollo tecnológico.

API 4. Reforzar el suministro privado de bienes y servicios básicos, las oportunidades de creación

7 Ver documento CA Sector Privado Asamblea de Gobernadores. de ingresos y la movilidad social para las poblaciones vulnerables.

API 5. Fomentar el crecimiento respetuoso del medioambiente.

Además, la herramienta estratégica que servirá de guía para originar los proyectos será el enfoque de cartera, vigente en la Cll desde 2007, según el cual las operaciones se analizan no sólo individualmente, sino también en función de su contribución a la cartera global.

En cuanto a las consideraciones sobre la propuesta organizacional, destacar que:

- La reforma propuesta mejora la manera en la que el grupo BID fomenta el desarrollo del sector privado, puesto que permite centrarse en las competencias básicas al tiempo de cumplir los mandatos de desarrollo, mitigar los riesgos operativos, centralizar el conocimiento y brindar oportunidades de carrera profesional más importantes que las que permite el actual arreglo fragmentario. La Nueva Corporación consolidaría en su seno a la actual CII, OMJ y SCF. En el caso del FOMIN, a solicitud del Comité de Donantes, se acordó que cualquier propuesta de integración del FOMIN requerirá la aprobación del Comité de Donantes y posterior aprobación del Directorio Ejecutivo de la Nueva Corporación. La estructura organizativa propuesta para la Nueva Corporación es congruente con los requisitos y el marco establecidos en el actual Convenio Constitutivo de la CII. Sin embargo no es el resultado de «elevar» las ventanillas sin garantía soberana existentes en el BID y simplemente trasladarlas a la actual CII para implementar la fusión hacia afuera. Destaca, por ejemplo, la importancia de una coordinación eficaz entre las operaciones con y sin garantía soberana e incluye numerosas recomendaciones de diseño en el fortalecimiento de esta coordinación y su incorporación en la forma de hacer negocios de la Nueva Corporación.

- La Nueva Corporación funcionará como una entidad multilateral autónoma y muchos de sus servicios internos serán suministrados por el BID, en virtud de los acuerdos de servicios que serán renovados. 
- Los receptores de las intervenciones de la Nueva Corporación serán principalmente empresas e intermediarios financieros, pero también está previsto la inclusión de entidades públicas que apoyen al sector privado de forma directa o indirecta 8 .

- Junto a los productos financieros, también se dispondrán de servicios de cooperación técnica con financiamiento no reembolsable y servicios remunerados de asesoría.

A partir de la consolidación de las actividades del sector privado, se esperan obtener beneficios operativos, a través de políticas, procedimientos y sistemas optimizados que la Administración prevé se traduzcan en ahorros reales de gastos administrativos así como beneficios cualitativos derivados de la mejora en la coordinación entre operaciones entre otros.

No obstante, también se han previsto, unos costes de transición relacionados con la implementación de la Nueva Corporación, estimados en 35,5 millones de USD para el plazo de un año. Las proyecciones financieras suponen que la Nueva Corporación reembolsará la parte correspondiente de estos costes al BID en cuotas anuales a partir de 2016.

\subsection{Proceso de capitalización de la Nueva Corporación}

En marzo de 2015, la administración del Banco presentó varios escenarios de capitalización de la Nueva Corporación, basados en tres parámetros principales: 7 años de contabilidad cruzada, 7 años para las aportaciones de capital fresco, y distintos niveles de transferencias desde el BID a la Nueva Corporación. La introducción de la contabilidad

\footnotetext{
8 Mientras que la financiación de las empresas propiedad del Estado y subsoberanas está restringida bajo el Convenio Constitutivo de la CII, el Directorio Ejecutivo de la CII ha interpretado esta disposición de manera que permita a la Cll otorgar financiación a empresas privadas indirectamente a través de este tipo de entidades. De ser proporcionada la financiación a empresas de propiedad estatal y/o subsoberanas para uso final por parte de dichas entidades, estos activos serían enteramente contabilizados utilizando el capital ordinario del BID o fondos de terceros.
}

cruzada y las transferencias de capital fueron demandadas por muchos de los países no regionales para disminuir el coste de la fusión y finalmente introducidas por la administración.

En el marco del BID, se ha estimado que la movilización de las ventanillas de SCF y OMJ a la Nueva Corporación, liberará capital. Los países prestatarios solicitaron que este capital liberado sirviera para respaldar mayores operaciones sin garantía soberana en el BID. No obstante, se ha decidido utilizar una parte del capital liberado para realizar las transferencias y el resto retenerlo para reforzar las ratios de capital del Banco.

\subsection{La Resolución de Busan}

Del 26 al 29 de marzo de 2015 ha tenido lugar en Busan, Corea del Sur, la Asamblea Anual del BID y de la CII. El punto principal de la agenda era aprobar la organización y la capitalización de la Nueva Corporación y también se aprobó la resolución que actualiza la estrategia institucional del Grupo BID para el periodo 2016-2020.

Respecto a la organización, se encomendó al Directorio Ejecutivo y a la administración del BID que adopten todas las medidas necesarias para realizar cualquier ajuste que sea necesario en la organización del BID que resulte de transferir las funciones operacionales y administrativas y los recursos no financieros vinculados a las actividades sin garantía soberana del BID a la CII.

La CII llevará a cabo toda la gama de operaciones que actualmente realicen las ventanillas sin garantía soberana del grupo del BID (incluyendo a las empresas de propiedad total del Estado y excluyendo las operaciones con Gobiernos subsoberanos). La Cll deberá tratar de mantener al menos su calificación de AA actual.

La resolución que establece el proceso de capitalización de la Nueva Corporación autoriza un aumento de capital de la Cll de 125.474 nuevas acciones con un precio base de 16.178,6 USD, es decir una ampliación de capital por un total de 2.030 millones de dólares. 


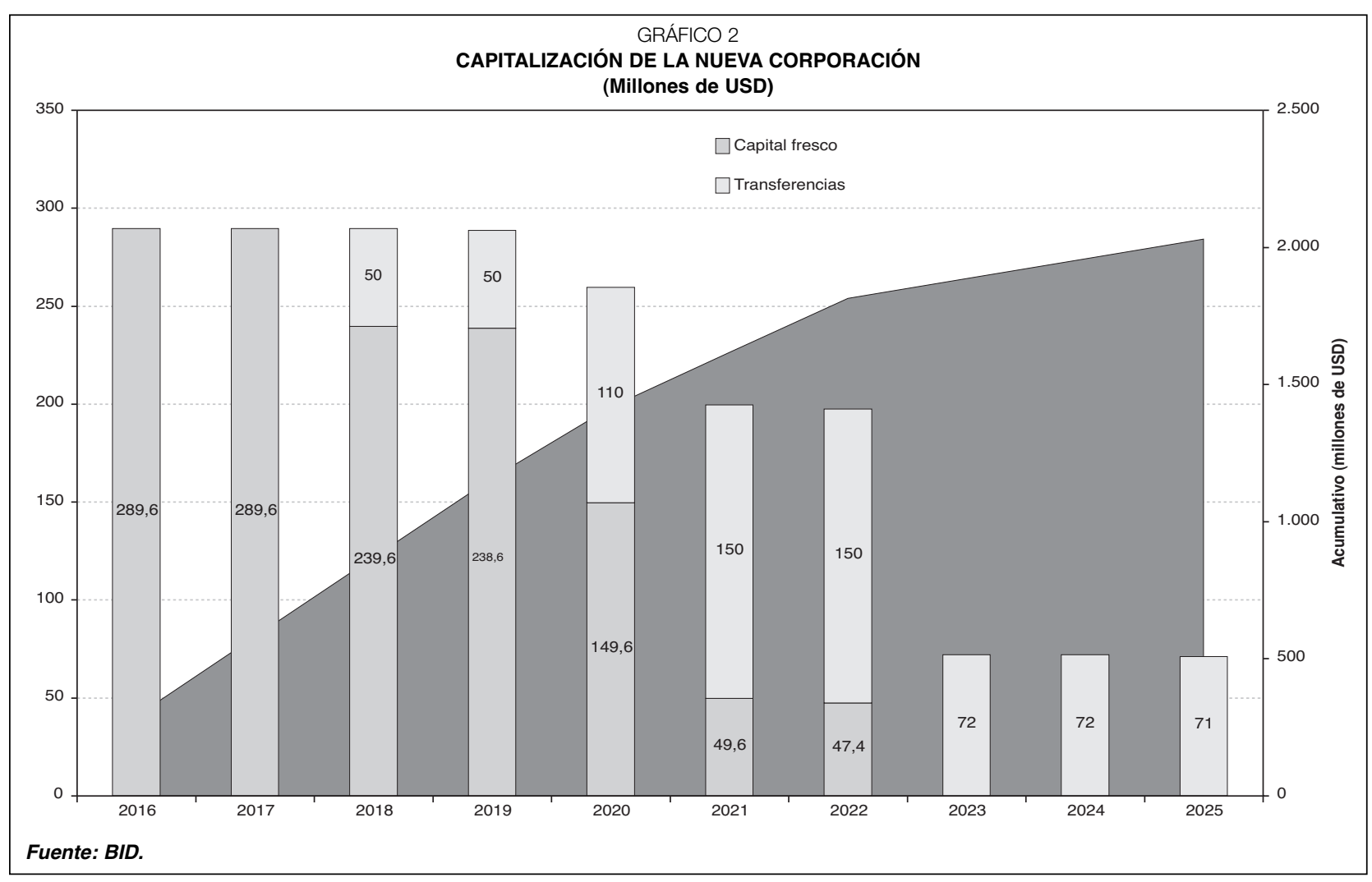

Las negociaciones respecto a cómo llegar a un acuerdo respecto a la capitalización de la Nueva Corporación ya se preveían complicadas y así fueron. De los 2.030 millones de dólares estos se dividen en:

- 1.305 millones de USD de capital fresco.

- 725 millones de USD de transferencias, de las cuales 215 millones se transferirán entre 2023 y 2025, sujetas al cumplimiento de la nueva política de adecuación de capital, incluída la plena constitución de franjas de capital, y siempre que se haya efectuado el pago de al menos el 50 por 100 de las nuevas acciones emitidas.

Cualesquiera nuevas acciones que no se suscriban o paguen de acuerdo con las condiciones establecidas, y cualequiesquiera nuevas acciones cuya suscripción se cancele, se reasignarán entre los accionistas de la Cll de conformidad con los términos y condiciones que establezca el Directorio Ejecutivo de la Cll, con el objetivo de asegurar la capitalización íntegra y oportuna.

Durante la Asamblea en Busan, solo España, China y Corea confirmaron públicamente la posibilidad de suscribir acciones en la nueva institución por encima de las que les correspondería. El resto de países mostraron su apoyo a la creación de la Nueva Corporación y en general se espera que suscriban al menos un número de acciones que les hagan mantener el mismo porcentaje de voto que en la actual CII (excepto EEUU que informalmente manifestó su imposibilidad de poder suscribir un aumento de capital en el corto plazo). También se espera que muchos países suscriban acciones adicionales, pero la magnitud dependerá principalmente de cual sea la oferta final de acciones sin suscribir.

España confirmó su apoyo a la Nueva Corporación y el interés en participar en el proceso de capitalización. En estos momentos, para mantener nuestra cuota actual en la CII (3,53 por 100), tendríamos que aportar capital fresco por valor de 45,81 millones de dólares en un período de 7 años (2016-2022).

\subsection{EI FOMIN}

En cuanto al FOMIN, uno de los puntos clave en este proceso va ser probablemente su encaje en $D$ 
la nueva estructura. Como ya hemos destacado, cualquier propuesta de integración del FOMIN requerirá la aprobación del Comité de Donantes y posterior aprobación del Directorio Ejecutivo de la Nueva Corporación. La discusión de la reposición del FOMIN y por lo tanto su futuro, ha estado ligada a lo largo de todo el proceso a la decisión sobre la reforma de la actividad del sector privado del Grupo BID. Esto ha llevado a que se aprobara el modelo de optimización de activos y liquidez así como las prácticas contables revisadas, que han dado tranquilidad al FOMIN para continuar con sus operaciones sin problemas hasta 2017, retrasando la necesidad de reposición $n^{9}$. El comité de donantes considerará en 2015 una serie de propuestas para el futuro de dicho fondo.

\section{Conclusión}

EI BID es una institución de referencia en la región de América Latina y el Caribe y consideramos que debería tener un papel más relevante

\footnotetext{
9 Sin embargo, no se descarta que se ponga en marcha NewCo antes de integrar el FOMIN, por lo que una mínima reposición para la continuidad del FOMIN podría ser solicitada ya en 2016.
}

en el apoyo del sector privado de la región. La consolidación de las diferentes ventanillas en una Nueva Corporación de apoyo al sector privado proporciona al grupo BID una mayor coherencia y eficacia en sus actuaciones para la promoción del desarrollo del sector privado y las operaciones sin garantía soberana.

España es país miembro fundador de la Cll y ha apoyado a esta institución, con buenos resultados desde su creación, tanto a través de la suscripción de capital accionarial como a través del Fondo Español de consultoría en el BID que apoya, sobre todo, el fomento de la competitividad de las pymes en la región.

Tras la aprobación de la Resolución del proceso de capitalización de la Nueva Institución, adoptada en la Asamblea de Corea en marzo de 2015, el verdadero reto comienza ahora. La administración del Banco está poniendo en marcha el proceso de transición con el objetivo principal de reforzar y optimizar los recursos hacia el sector privado a la vez que se mantiene el nivel y calidad de las operaciones con garantía soberana.

Está demostrado que mejorar la baja productividad en América Latina y el Caribe es un desafío y que contar con el sector privado como importante agente del desarrollo es fundamental. 


\section{NORMAS DE ESTILO DE PUBLICACIÓN}

La persona o personas interesadas en presentar un artículo para su publicación en el Boletín de Información Comercial Española (BICE) deberán enviar el artículo en formato Microsoft Word a la dirección de correo electrónico revistasice.sscc@comercio.mineco.es

El documento debe cumplir las siguientes características:

1. Ser material original no publicado ni presentado en otro medio de difusión.

2. La extensión total del trabajo (incluyendo cuadros, gráficos, tablas, notas, etcétera) no debe ser inferior a 15 páginas ni superior a 20. La fuente será Times New Roman, tamaño 12 y espaciado doble. Estar paginado en la parte inferior derecha de cada página.

3. En la primera página se hará constar el título del artículo que deberá ser breve, claro, preciso e informativo y la fecha de conclusión del mismo. Nombre y dos apellidos del autor o autores, filiación institucional, dirección, teléfono y correo electrónico de cada uno de ellos, así como la forma en que desean que sus datos aparezcan.

4. En la segunda página del texto se incluirá:

- El título.

- Un resumen del trabajo con una extensión máxima de 10 líneas con la siguiente estructura: objetivo, método y principal resultado o conclusión.

- De 2 a 6 palabras clave que no sean coincidentes con el título.

- De 1 a 5 códigos de materias del Journal of Economic Literatura (clasificación JEL) para lo cual pueden acceder a la siguiente dirección electrónica:

https://www. aeaweb.org/jel/guide/jel.php

5. En las siguientes páginas se incluirán el texto, la información gráfica y la bibliografía con la siguiente organización.

- Se incluirá, por este orden, introducción, desarrollo, conclusiones y bibliografía y anexos si los hubiera. Los apartados y subapartados se numerarán en arábigos respondiendo a una sucesión continuada utilizando un punto para separar los niveles de división, según el siguiente modelo:

\section{Título del apartado}

\subsection{Título del apartado}

\subsubsection{Título del apartado}

\section{Título del apartado}

- Las notas de pie de página irán integradas en el texto y su contenido debe estar al final de su misma página en tamaño 10 y espacio sencillo.

- En un archivo Excel independiente se incluirá la representación gráfica (cuadros, gráficos, diagramas, figuras, etc.), que debe llevar título, estar numerada y referenciada en el texto. En la parte inferior se incluirá la fuente de información y/o notas aclaratorias.

- Las citas de libros y artículos en el texto, se indicarán entre paréntesis con el apellido del autor y el año. Ej.: (Martínez, 1991).

- Las referencias a siglas deben ir acompañadas, en la primera ocasión en que se citen, de su significado completo.

- La bibliografía se ordenará alfabéticamente siguiendo las normas de la American Psychological Association (Harvard-APA): http://cibem.org/paginas/img/apa6.pdf

Libros

APELLIDOS, A.A. (año de publicación). Título del libro (edición) (volumen). Ciudad: Editorial. Artículo en revista científica

APELLIDOS, A.A. (año de publicación). «Título del artículo». Título de la revista, volumen (número), números de páginas.

Documento en línea

APELLIDOS, A. A. u ORGANISMO (año, mes de publicación). Título, [en línea]. Ciudad: Editorial. Disponible en:

http://cenamb.rect.ucv.ve/siamaz/dicciona/canaima/canaima2.htm [Recuperado: 2000, 3 de junio]. 